\title{
Correction to: Converting STEM into STEAM Programs
}

\author{
Arthur J. Stewart, Michael P. Mueller, and Deborah J. Tippins
}

\section{Correction to:}

\section{A. J. Stewart et al. (eds.), Converting STEM into STEAM Programs, Environmental Discourses in Science Education 5, https://doi.org/10.1007/978-3-030-25101-7}

In the original version of the book, the following correction has been incorporated: The chapter author's, "Sophia (Sun Kyung) Jeong", first and last names have been corrected. The correction chapter and book have been updated with the change.

The updated version of these chapters can be found at 